\title{
La independización y el empoderamiento del estudiante como actitudes necesarias en la implementación de las adecuaciones curriculares
}

\author{
M. Ed. José Pablo Bolaños Segnini
}

Gracias al esfuerzo de la Humanidad por acelerar el desarrollo de su conocimiento en todos los campos y sus capacidades tecnológicas, las tecnologías de comunicación han experimentado una asombrosa diversificación y expansión en el último siglo. Esto ha facilitado no sólo el intercambio de conocimientos entre diversos sectores de la población mundial, sino la colaboración para el desarrollo de complejas relaciones comerciales, a pesar de la diversidad de culturas y condiciones sociales que caracterizan a dicha población. El desarrollo tecnológico, entonces, ha estimulado la interacción y negociación entre distintos grupos humanos. En este contexto, resulta esencial mantener presente que el Ser Humano, como eje fundamental de este escenario, ha demostrado una tendencia a establecer relaciones que no siempre favorecen en igual forma y medida a los distintos individuos o grupos involucrados. 
El autor Edgar Morín (2000) señala que las mismas características de nuestro sistema productivo que impulsan la modernización también incrementan las desigualdades entre distintos actores sociales, inoculando con ello sentimientos de inseguridad y motivando reacciones compulsivamente defensivas ante todo lo desconocido y lo diverso. Al tomar en cuenta los derechos fundamentales y la dignidad de la persona humana, resulta imperativo establecer mecanismos para velar porque haya equidad para todos los actores sociales en el mejoramiento del nivel de vida en un concepto amplio de libertad y en el progreso social. Es a partir de esta concepción que se ha establecido la importancia del análisis de las diversas necesidades del individuo para que goce de todas las oportunidades de acceso y participación en distintos ámbitos de la vida comunitaria que le confieren los derechos humanos y las garantías sociales.

Todo este reconocimiento tiene un carácter universal que se respalda con una normativa jurídica internacional, que incluye la Declaración de Derechos Humanos y la Convención de Derechos del Niño de las Naciones Unidas, y propia de cada país. Para el caso específico de Costa Rica, dicha normativa jurídica incluye la Constitución Política y la Ley 7600 de Igualdad de Oportunidades para las Personas con Discapacidad, así como en las normativas específicas del sistema educativo costarricense, tanto para la prática pedagógica como para la evaluación de los aprendizajes.

La sociedad mundial se debate entre la tolerancia a las diferencias y la aceptación de la diversidad, que son dos posiciones profundamente distintas. En la primera, el término diferencias se refiere las desigualdades, que lleva implícitas la inequidad social y la marginación de ciertos grupos; la resolución de estas desigualdades sucede por medio de una imposición de formas de pensar y actuar, una "licuación de dichas diferencias". Es una postura que está enmarcada en un 
discurso de carácter hegemónico. En la segunda posición, diversidad se refiere a la existencia de múltiples culturas y necesidades, formas de pensar y sentir, identidades que tienen un valor propio. Implica el reconocimiento de la necesidad de un proceso de diálogo por medio del cual se sienten las pautas para lograr una ințegración (Díaz \& Alonso, 1998).

La misma situación que se presenta en la sociedad, se ha reproducido - con ciertas especificidades del contexto pedagógico - en la escuela. En el medio educativo, se llegó a considerar como normal lo que no lo era: la desigualdad que domina en la vida y en la sociedad (Morín, 2000). Sin embargo, el desarrollo de un enfoque de atención a la diversidad en los últimos veinte años, ha motivado esfuerzos para la elaboración de proyectos educativos que contribuyan a reconocer y suprimir las desigualdades, en vez de reproducirlas o aumentarlas.

De ahí, que una de las características fundamentales del modelo de atención a la diversidad en educación es, que insiste en la adaptación del tratamiento pedagógico a las necesidades específicas de cada uno de sus estudiantes, puesto que la eficacia de un centro educativo depende de su capacidad para realizar los ajustes internos necesarios para que todos los estudiantes alcancen los objetivos propuestos (Pazos, S.F.). Estos ajustes, por supuesto, van desde la infraestructura (en el ámbito material) y la estructura (en el ámbito organizativo) hasta el currículo y modelo pedagógico (en el ámbito académico).

El Ministerio de Educación Pública, como organismo ejecutor de la normativa jurídica en el campo de la educación, es el responsable de emitir las directrices correspondientes a la atención pedagógica en general y, más específicamente, de los individuos que, en virtud de sus características y necesidades educativas, requieran de ajustes en la propuesta curricular o metodológica. De ahí surge 
la propuesta de una serie de estrategias para la integración de estudiantes con diversidad de necesidades educativas que se conocen como adecuaciones curriculares.

El Ministerio de Educación Pública define las adecuaciones curriculares como las acciones que realiza el docente para ajustar la programación educativa y ofrecer experiencias apropiadas que atiendan las necesidades particulares de cada estudiante (MEP, 1998). Pueden incluir ajustes en el planeamiento metodológico para crear situaciones de aprendizaje o de evaluación adecuadas (adecuaciones curriculares no significativas), modificaciones a los objetivos y/o contenidos del currículo (adecuaciones curriculares significativas), y el uso de recursos materiales o tecnológicos especiales (adecuaciones curriculares de acceso). Los objetivos explícitos de las adecuaciones curriculares son, por supuesto, facilitar la participación de todos los estudiantes, independientemente de las necesidades especiales en los ambientes de aprendizaje, para que sean capaces de cumplir los objetivos de aprendizaje propuestos. Es decir, para que todos los estudiantes tengan igual oportunidad de desarrollar y potenciar sus capacidades dentro de nuestro sistema educativo.

La experiencia práctica en la aplicación de las adecuaciones curriculares - que son la estrategia que el sistema educativo nacional ha propuesto para la integración de estudiantes con diversidad de necesidades educativas - revela que existen aún numerosos obstáculos que deben superarse antes de poder construir un sistema educativo que sea realmente integrador. Estas barreras incluyen la escasa capacitación del personal docente para la atención de la diversidad de necesidades educativas especiales, la existencia de confusiones en torno al concepto de las adecuaciones curriculares, el surgimiento de prejuicios negativos y barreras actitudinales en contra de las adecuaciones curriculares. A 
continuación, describo un problema que frecuentemente he enfrentado en la aplicación de las adecuaciones curriculares y que está asociado a la manera en que se entienden los alcances y los límites de esta estrategia.

Una parte importante de la función del educador está relacionada con la capacidad para ayudar al estudiante a desarrollar actitudes y capacidades que propicien su adaptación al medio en que se desenvuelve, su desempeño exitoso dentro del mismo. De hecho, la existencia de tecnologías capaces de transmitir todo tipo de información y ayudar al estudiante en la construcción de nuevos conceptos, ha motivado una reflexión en el medio académico sobre la función que realmente justifica la existencia del educador. Dicha función no es la de transmitir ideas sino la de ayudar a los estudiantes a ser independientes y adquirir hábitos de organización, trabajo y estudio; a desarrollar actitudes y habilidades que le permitan resolver sus problemas de manera autónoma en colaboración con los demás miembros de la comunidad escolar. Desdichadamente, esta función es frecuentemente obviada en la educación por razones diversas, especialmente cuando se trabaja con estudiantes que han sido diagnosticados con algún tipo de problema de aprendizaje.

El caso específico de Andrés ${ }^{1}$ permite ejemplificar esta situación. Él es un estudiante de once años de edad y a pesar de haber avanzado mucho en el manejo y el control de su condición, aún escribe muy despacio. Por lo tanto, una de las adecuaciones curriculares que se sugieren en el caso de Andrés es el uso de materiales poligrafiados/fotocopiados para prevenir que se atrase innecesariamente en el desarrollo del trabajo en clase por tener que copiar las instrucciones de la

\footnotetext{
${ }^{1}$ Aunque el caso es real, el nombre y algunos otros datos han sido modificados para resguardar la privacidad del estudiante y de su familia.
} 
pizarra. La madre se presenta a una reunión del Comité de Apoyo para discutir los alcances que debe tener dicha estrategia. Ella insiste en que el estudiante no debería copiar del todo e incluso llega al punto de sugerir que sean los mismos docentes quienes dejen copia escrita en la agenda del muchacho de las asignaciones (tareas, trabajos, y demás) que van quedando a lo largo del tiempo. La reacción de los miembros del personal docente que están presentes en la reunión, es de indignación. Como Psicólogo de una institución educativa privada, esta situación demanda una complicada mediación entre la madre y los docentes para llegar a compromisos benéficos para el estudiante. Aunque el conflicto es resuelto en este caso, el problema es que este tipo de situaciones no representa la excepción; antes bien, son bastante comunes y no todas las instituciones educativas de nuestro país cuentan con las condiciones y con el personal para resolver eficazmente este tipo de conflictos.

La sobreprotección es el problema de crianza más común que puede ser observado en las familias de personas que han sido diagnosticadas con algún tipo de discapacidad, malformación, enfermedad crónica, psicopatología o problema de aprendizaje, entre otros. La sobreprotección se manifiesta en el estudiante de varias formas, incluyendo baja autoestima, baja confianza propia, dependencia excesiva del docente o de los compañeros para el cumplimiento del trabajo asignado, hábitos de organización y de trabajo pobremente desarrollados, ansiedad excesiva en situaciones donde se siente evaluado y, en algunos casos, la presencia de estrategias bien desarrolladas de manipulación.

En el caso de Andrés, la sobreprotección de su madre se manifiesta de todas las formas ya mencionadas y los docentes se han percatado de ello. La madre asume como un derecho de Andrés, en acuerdo con la Ley 7600 sobre Igualdad de Oportunidades para las Personas con Discapacidad, 
el hecho de contar con material poligrafiado/fotocopiado en cualquier situación en que sea necesario copiar o tomar nota de algo (particularmente si es extenso y si eso compromete en cualquier medida su capacidad para concluir el trabajo en clase, en igualdad de condiciones que el resto de sus compañeros). Los docentes asumen como parte de su función someter a Andrés a un proceso de entrenamiento para que vaya siendo capaz de tomar nota de las cosas que resultan necesarias en el medio escolar y, por qué no, llegar algún día incluso a prescindir por completo del material poligrafiado/fotocopiado. Tanto la madre como los docentes, tienen razón en alguna medida.

Más allá de los aspectos prácticos de la discusión, que pueden ser resueltos de múltiples formas y - de hecho fueron resueltos sin gran dificultad, surgen en esta situación una serie de cuestionamientos que valdría la pena someter a discusión. Por ejemplo, ¿resulta normal y deseable que un estudiante con adecuación curricular haga un esfuerzo por adaptarse a las condiciones del medio escolar?, ¿quién puede realmente determinar hasta dónde llegan las capacidades de un estudiante con adecuación curricular para adaptarse a su medio mediante sus propias capacidades y para superar sus limitaciones?, ,hasta dónde es deseable (o posible) que el sistema educativo (o el medio en general) se ajuste a las necesidades (y los déficits) de un estudiante cualquiera?

El siguiente ejemplo resulta útil para buscar respuesta a estas preguntas. Valeria ${ }^{*}$ es una estudiante de doce años de edad con severos problemas de epilepsia convulsiva (grand mal) que fue, además, diagnosticada con un retraso general de su desarrollo intelectual, el cual estaría presuntamente asociado al deterioro de sus capacidades cognoscitivas resultante de su condición. Paralelamente, la estudiante ha sido diagnosticada con Síndrome de Déficit Atencional. Cuando ingresó a la institución unos cuatro 
años atrás, se mostraba incapaz de realizar y concluir las mismas evaluaciones escritas que el resto de sus compañeros y, por sugerencia de una psicopedagoga que ha dado seguimiento privado al caso, se le han realizado pruebas específicas desde el primer momento. La docente que está a cargo de Valeria, por su parte, ha insistido en ir disminuyendo de manera gradual la cantidad de modificaciones que realiza a las pruebas de la niña e ir aproximando cada vez más dichas pruebas específicas a las pruebas regulares que se aplican al resto de los estudiantes. El desempeño de la niña ha sido favorable a lo largo del tiempo, a pesar de las protestas que inicialmente presentara su psicopedagoga. Más importante aún, los niveles de frustración y agresividad de la niña han disminuido, y todo parece indicar que su autoestima y su nivel de confianza en sí misma van en aumento.

Diagnósticos como los de Andrés (dislexia) y Valeria (retardo mental) deben ser entendidos como fotografías que se toman del funcionamiento de un individuo en un campo específico y un momento específicos. Es un error obviar el fenómeno común del sobrediagnóstico o asumir cualquier diagnóstico como una verdad absoluta, incuestionable y permanente, porque la mayoría de las personas con este tipo de diagnósticos han demostrado que pueden ser entrenados para superar muchas de las limitaciones que les fueron inicialmente diagnosticadas. Muchos estudiantes diagnosticados con alguna variedad del Síndrome de Déficit Atencional han demostrado que pueden ser entrenados para aprender a controlar mejor sus impulsos y para ampliar los periodos en que pueden mantener enfocada su atención en una tarea. Estudiantes diagnosticados con Síndrome de Asperger, han demostrado que pueden llegar a desarrollar, a través del tiempo, estrategias conductuales para controlar su conducta ansiosa y compulsiva, hacerse entender e interactuar más eficazmente, tanto con sus compañeros como con sus docentes. 
Tenemos, por un lado, toda una normativa jurídica que garantiza a todos los estudiantes que el sistema educativo realizará los ajustes necesarios para permitirles cumplir los objetivos de aprendizaje que han sido planteados para ellos. Por otro lado, la experiencia acumulada con estudiantes de adecuación curricular demuestra que muchos estudiantes pueden llegar a compensar sus limitaciones y disminuir la necesidad de dichos ajustes, siempre y cuando, logren antes superar los obstáculos que afrontan en el plano emocional y actitudinal.

Por supuesto, lograr que un estudiante con adecuación curricular alcance un grado de independencia en su entorno educativo y familiar, no es una labor sencilla. Requiere de un plan desarrollado de manera colectiva, de un abordaje adaptado a cada caso particular y de mucho tiempo para valorar los resultados en el ámbito actitudinal del estudiante. Como punto de partida, sin embargo, es posible recomendar algunas estrategias que puedan fomentar la independencia en alumnos con todo tipo de necesidades educativas. A saber:

- El manejo de un organizador personal y bitácora por parte del estudiante. Es una experiencia que brinda al estudiante la oportunidad de llevar un control independiente de sus obligaciones escolares y administrar su propio tiempo. Si los padres de familia pueden llegar a limitar al estudiante en la posibilidad de llevar ese control, como el correo electrónico o el cuaderno de mensajes, para centralizar este control, entonces limitan la posibilidad del estudiante de responsabilizarse de sus obligaciones escolares. En realidad, el manejo de una agenda es una destreza que sólo una minoría de estudiantes podría tener dificultades para desarrollarla. 
- El desarrollo de hábitos de estudio independiente. Inicia con el desarrollo de un horario de estudio que el estudiante puede cumplir con un mínimo de control cruzado por parte de la familia y del personal docente. La experiencia demuestra que muchos estudiantes con adecuación curricular no son capaces de estudiar o cumplir con sus tareas si no cuentan con el acompañamiento inmediato de un familiar o tutor que les ayude. Muchas familias incluso llegan a considerar normal que un estudiante sea incapaz de realizar un esfuerzo propio por cumplir con una labor si no cuentan con la presencia de un tutor. Sin embargo, la experiencia en la elaboración de resúmenes, el uso de técnicas de aprendizaje visual como mapas conceptuales, el uso de técnicas de aprendizaje auditivo como la grabadora y los repasos verbales, entre otras, puede permitir al estudiante ir conociendo mejor cómo puede potenciar su propio aprendizaje.

- El desarrollo de destrezas sociales. Las habilidades para comunicarse, para negociar y resolver problemas son destrezas esenciales para una buena adaptación en todos los ámbitos de la vida social. La experiencia de dialogar abiertamente con los docentes, solicitar ayuda cuando considera que la necesita, discutir las adecuaciones curriculares, negociar espacios cuando resulte pertinente, entre otras, puede ayudar al estudiante a desarrollar una mayor autoestima y confianza en sí mismo, así como una mayor capacidad de autogestión, para dejar atrás la sensación de minusvalía y desmotivación que a veces le aqueja.

A menudo, los padres de los estudiantes con adecuación curricular tratan de asumir el papel de interlocutores e intermediarios de sus hijos, lesionando de esa forma las posibilidades de desarrollo del estudiante. 
La escuela para padres es un espacio que manejan muchas instituciones, en el cual la sobreprotección y otros problemas familiares que afectan el rendimiento de los estudiantes pueden ser analizados, y puede ofrecerse entrenamiento para que dichos problemas sean superados.

- El desarrollo de una actitud preactiva o "proactiva". Significa la disciplina del estudiante de sacar provecho de todas las opciones de apoyo que se le ofrecen en el medio escolar. En una ocasión, la directora de un centro educativo me comentaba que "sus docentes tienen dificultad para comulgar con la aplicación de adecuaciones curriculares cuando los estudiantes no cumplen plenamente con todo su trabajo y no parecen sacar pleno provecho de las oportunidades de apoyo que se les brindan". En ocasiones, los padres de familia y los mismos estudiantes tienen como expectativa que el estudiante con adecuación curricular será capaz de lograr el éxito académico independientemente de su grado de esfuerzo y aprovechamiento en el trabajo escolar.

Se conocen casos, por ejemplo, en que los docentes han brindado la oportunidad a todo un grupo de estudiantes de traer corregidos los errores que cometieron en una evaluación escrita, con la intención de que aprendan de sus errores y puedan obtener algunos puntos adicionales en la evaluación. Sin embargo, hay estudiantes de adecuación curricular que, a pesar de estar entre quienes más necesitan una oportunidad de este tipo, no la aprovechan. Es esencial que los estudiantes conozcan cuáles son los apoyos didácticos y las opciones para mejorar sus calificaciones que la institución les brinda, para que puedan elegir así obtener total provecho. 
Todas las acciones anteriormente mencionadas comparten dos denominadores comunes. El primero es que tienen por fin último fomentar un desempeño más independiente en los estudiantes con adecuación curricular, un mayor dominio de cara a sus propias necesidades y sus propios procesos de aprendizaje. El segundo, que son algunas que más objetan los padres de estos estudiantes, precisamente por la percepción distorsionada que estos padres de familia (e incluso algunos educadores) tienen de los fines de las adecuaciones curriculares.

La atención a la diversidad es un imperativo para cualquier sistema educativo hoy día, pero las adecuaciones curriculares como principal estrategia para lograr esta atención deben cumplir con las mismas condiciones que requieren todas las demás estrategias didácticas. Deben constituirse verdaderas herramientas de apoyo, según la necesidad educativa que presenta el estudiante y deben ser formuladas en estrecha relación entre el docente (entendido como el especialista de la materia) y el alumno, que requiere llegar a la zona de desarrollo próximo (adquirir conocimiento y aprendizaje) con la ayuda del docente. Además, deben entenderse como decisiones y acciones específicas que no pueden estar desligadas de las demás decisiones y acciones pedagógicas más generales.

Por ejemplo, si suponemos que uno de los objetivos didácticos planteados para toda la población estudiantil en una institución cualquiera es que sean capaces de constituirse en estudiantes activos en su propio proceso de aprendizaje y en miembros proactivos de una comunidad, capaces de autogestionarse, no se podría permitir que los ajustes realizados para ayudar al estudiante lo conviertan en un estudiante receptivo y pasivo, limitando entonces la posibilidad de ese estudiante de cumplir con el objetivo propuesto.

Además, las adecuaciones curriculares deben ser comprendidas como medidas que conllevan una responsabilidad 
tripartita: de la institución, de la familia que apoya al estudiante $y$, principalmente, del estudiante que es el actor del proceso. Las adecuaciones curriculares no deben ser entendidas como una estrategia que exime de responsabilidad al estudiante por el simple hecho de que demanda un ajuste por parte de la institución. Esta adaptación necesaria para una debida atención de la diversidad de necesidades que se presentan en la comunidad estudiantil, tiene por objetivo permitir al estudiante las mismas posibilidades que disfrutan los demás estudiantes de integrarse exitosamente al sistema educativo y de cumplir con los objetivos de aprendizaje requerido. Morín (2000) explica que "se trata de favorecer la igualdad de oportunidades y la comprensión de las desigualdades mediante una enseñanza comprensiva e inclusiva, como garante de la atención a la diversidad en sus más variadas modalidades y expresiones". Garantizar esta posibilidad, no implica de ninguna manera eximir a un estudiante de las exigencias y responsabilidades que pesan sobre el resto de la sociedad.

Si entendemos que un sector de la sociedad globalizada considera como normal que existan mecanismos de marginación, podemos suponer que el sistema educativo, a la par de la responsabilidad por superar esta visión de la realidad para lograr un sistema más justo para todos los estudiante, también afronta la responsabilidad de empoderar a todos esos estudiantes para que tengan éxito dentro de una sociedad que presenta esas características. En ese contexto, la única acción responsable por parte de cualquier docente es preparar a un estudiante para que sea cada vez más independiente, para que se valga por sí mismo, para que comprenda las bases ideosincrásicas y los mecanismos de la marginación, de la discriminación y la intolerancia. Consecuentemente, el ejercicio de esta práctica pedagógica formará al estudiante para que sea capaz de defender sus derechos en 
un marco de respeto, de abogar por una sociedad más justa para sí mismo y para las personas que tienen características similares a las suyas.

\section{REFERENCIAS BIBLIOGRÁFICAS}

Díaz, Raúl \& Alonso, Graciela (1998). La Dimensión "mundo" de la globalización y las identidades culturales. (En Red: http:// www.naya.org.ar/congreso/ponencial-5.htm)

Morín, Edgar (2000). La Cabeza Bien Puesta. Pensar la Reforma, Reformar el Pensamiento. Editorial Nueva Visión: Buenos Aires.

Pazos, Ethel (S.F.). La Necesidad de un Nuevo Modelo Educativo de Atención a la Diversidad. 